\title{
Cognitive performance and psychosocial functioning in patients with bipolar disorder, unaffected siblings, and healthy controls
}

\author{
Mirela P. Vasconcelos-Moreno, ${ }^{1,2}$ Joana Bücker, ${ }^{1,2}$ Kelen P. Bürke, ${ }^{1,2}$ Leticia Czepielewski, ${ }^{1,2}$ \\ Barbara T. Santos, ${ }^{1}$ Adam Fijtman, ${ }^{1}$ Ives C. Passos, ${ }^{1,2}$ Mauricio Kunz, ${ }^{1,2}$ Caterina del Mar Bonnín, ${ }^{3}$ \\ Eduard Vieta, ${ }^{3}$ Flavio Kapczinski, ${ }^{1,2}$ Adriane R. Rosa, ${ }^{1,2}$ Marcia Kauer-Sant'Anna ${ }^{1,2}$ \\ ${ }^{1}$ Programa de Transtorno Bipolar, Laboratório de Psiquiatria Molecular, Instituto Nacional de Ciência e Tecnologia Translacional em Medicina \\ (INCT-TM), Hospital de Clínicas de Porto Alegre (HCPA), Universidade Federal do Rio Grande do Sul (UFRGS), Porto Alegre, RS, Brazil. \\ ${ }^{2}$ Programa de Pós-Graduação em Psiquiatria, UFRGS, Porto Alegre, RS, Brazil. ${ }^{3}$ Bipolar Disorders Program, Institute of Neurosciences, \\ Hospital Clinic, University of Barcelona, Institute of Biomedical Research August Pi i Sunyer (IDIBAPS), Biomedical Research Networking \\ Center for Mental Health Network (CIBERSAM), Barcelona, Catalonia, Spain.
}

\begin{abstract}
Objective: To assess cognitive performance and psychosocial functioning in patients with bipolar disorder (BD), in unaffected siblings, and in healthy controls.

Methods: Subjects were patients with BD $(n=36)$, unaffected siblings $(n=35)$, and healthy controls $(n=44)$. Psychosocial functioning was accessed using the Functioning Assessment Short Test (FAST). A sub-group of patients with BD ( $n=21)$, unaffected siblings $(n=14)$, and healthy controls $(n=22)$ also underwent a battery of neuropsychological tests: California Verbal Learning Test (CVLT), Stroop Color and Word Test, and Wisconsin Card Sorting Test (WCST). Clinical and sociodemographic characteristics were analyzed using one-way analysis of variance or the chi-square test; multivariate analysis of covariance was used to examine differences in neuropsychological variables.

Results: Patients with BD showed higher FAST total scores $(23.90 \pm 11.35)$ than healthy controls $(5.86 \pm 5.47 ; p<0.001)$ and siblings $(12.60 \pm 11.83 ; p$ 0.001). Siblings and healthy controls also showed statistically significant differences in FAST total scores $(p=0.008)$. Patients performed worse than healthy controls on all CVLT sub-tests $(p<0.030)$ and in the number of correctly completed categories on WCST $(p=0.030)$. Siblings did not differ from healthy controls in cognitive tests.

Conclusion: Unaffected siblings of patients with BD may show poorer functional performance compared to healthy controls. FAST scores may contribute to the development of markers of vulnerability and endophenotypic traits in at-risk populations.
\end{abstract}

Keywords: Bipolar disorder; siblings; functional impairment; cognition; endophenotype

\section{Introduction}

The high heritability of bipolar disorder (BD) has been demonstrated in several twin and adoption studies. ${ }^{1}$ The risk of first-degree relatives developing $B D$ is approximately 10 -fold higher than that of the general population. ${ }^{2}$ Despite the evidence supporting the existence of neurobiological trait abnormalities in individuals at genetic risk for BD, most individuals with a family history of BD will not develop the disorder. ${ }^{2}$ Conversely, they may present subtle symptoms or vulnerabilities, which are less studied.

$\mathrm{BD}$ is associated with psychosocial functioning decline, and greater impairment is commonly observed at later stages of the illness. ${ }^{3}$ Functional disabilities in BD are not limited to symptomatic periods; they may remain present

Correspondence: Mirela Paiva Vasconcelos Moreno, Laboratório de Psiquiatria Molecular, Instituto Nacional de Ciência e Tecnologia Translacional em Medicina (INCT-TM), Hospital de Clínicas de Porto Alegre, Universidade Federal do Rio Grande do Sul, Rua Ramiro Barcelos, 2350, CEP 90035-903, Porto Alegre, RS, Brazil.

E-mail: mpvasconcelosmoreno@gmail.com

Submitted Nov 26 2015, accepted Dec 162015. or result in sustained disability. ${ }^{4}$ In a follow-up study, after two years, $98 \%$ of first-episode manic patients had symptomatic recovery, whereas only $38 \%$ had functional recovery. ${ }^{5}$ Therefore, the inability of patients with $\mathrm{BD}$ to function socially and occupationally and to live independently is not necessarily associated with symptom status. $^{5}$

Neurocognitive deficits have been consistently correlated with functional impairment in BD and have been pointed out as one of the most relevant predictors of functionality in $\mathrm{BD} .^{6-8}$ Patients with poor functioning have been shown to present poorer cognitive performance, particularly in verbal memory and executive function, even when euthymic. ${ }^{6,7,9-11}$ Forcada et al. ${ }^{8}$ emphasized that intelligence quotient $(\mathrm{IQ})$, total white matter volume, and course of depressive illness were independently associated with functional outcome in patients (assessed with the Global Assessment of Functioning Scale), but not in relatives (siblings and offspring). Studies with firstepisode manic patients have consistently shown deficits in visual, verbal, and working memory when compared to healthy controls. ${ }^{12,13}$ 
Some studies have suggested the presence of cognitive dysfunction in unaffected first-degree relatives of patients with $\mathrm{BD}$ when compared to healthy controls, particularly in executive function and/or verbal memory. ${ }^{14-16}$ Arts et al. ${ }^{14}$ in a meta-analysis, showed a small effect size $(d<0.500)$ for executive function and verbal memory parameters in first-degree relatives.

Moreover, there is evidence supporting the existence of neurobiological trait abnormalities in individuals at genetic risk for $\mathrm{BD} .{ }^{17} \mathrm{~A}$ systematic review of neuroimaging studies involving individuals at high genetic risk for BD (twins, siblings, offspring, first-degree, and second-degree relatives of patients) found accumulating structural and functional imaging evidence suggesting abnormalities in this population. ${ }^{17}$

Even though $\mathrm{BD}$ has a strong genetic underpinning, little is known about clinical or biological features associated with risk for the illness. In this sense, a better understanding of the familial risk for BD in siblings and of how this familial risk is clinically manifested could help researchers identify heritable sub-phenotypes of the disorder, as this population has high genetic loading for BD because of the shared DNA. It may also shed light on the pathophysiology of the condition, and possibly encourage further research into the genetic architecture of $\mathrm{BD}$.

Therefore, the objective of this study was to investigate the presence of neurocognitive and functioning impairment in patients with $\mathrm{BD}$, in unaffected siblings, and in healthy controls, comparing siblings to the two other groups, in an attempt to characterize neuropsychological performance and psychosocial functioning in a Brazilian population at genetic risk of developing $\mathrm{BD}$. Considering that cognitive deficits are one of the most relevant predictors of functioning in $\mathrm{BD}$, we thought it would be interesting to asses cognitive performance and functioning in unaffected siblings. We hypothesized that patients with $\mathrm{BD}$ and unaffected siblings would show impaired performance on cognitive and psychosocial functioning tests when compared with healthy controls.

\section{Methods}

\section{Participants}

This is a cross-sectional study reporting data obtained at the Bipolar Disorder Program, at Hospital de Clínicas de Porto Alegre (HCPA), Universidade Federal do Rio Grande do Sul, southern Brazil. Participants included outpatients receiving a diagnosis of $\mathrm{BD}$ type $\mathrm{I}$ at the program, one or more unaffected siblings of each patient included, and healthy controls. Thirty-six patients with BD, 39 unaffected siblings, and 44 healthy subjects were included. All patients were tested for mood symptoms and psychosocial functioning; a subgroup of 21 patients, 14 siblings, and 22 healthy controls underwent a battery of neuropsychological tests. Inclusion criteria for patients were: having a diagnosis of BD type I according to the Structured Clinical Interview for DSM-IV Axis I Disorders (SCID-I), being on current pharmacological treatment, being euthymic for at least 45 days, presenting scores $\leqslant 7$ on the Hamilton Depression Rating Scale (HDRS). and the Young Mania Rating Scale (YMRS), not having neurological diseases or severe clinical illnesses, having siblings without a diagnosis of $\mathrm{BD}$, and agreeing to participate in the study.

Siblings were included if they did not have a psychiatric diagnosis according to DSM-IV, if they scored $\leqslant 7$ on HDRS and YMRS, did not have neurological diseases or severe clinical illnesses, and if they agreed to participate in the study.

Finally, controls were volunteer blood donors invited and recruited at the Blood Bank of the HCPA. Inclusion criteria for the control group were: not having a psychiatric diagnosis according to DSM-IV, scoring $\leqslant 7$ on HDRS and YMRS, not having neurological diseases or severe clinical illnesses, having a negative psychiatric history, having a negative family history of psychiatric disorders (considering all first degree-relatives), and agreeing to participate in the study. Individuals with unstable inflammatory diseases were excluded. All controls underwent a comprehensive clinical interview conducted by a well-trained psychiatrist, using a short version of the SCID-I.

All the procedures described in this study were approved by the local Research Ethics Committees. Written informed consent was obtained from all the participants prior to any study procedures.

\section{Assessment}

Depressive and manic symptoms were assessed using HDRS and YMRS, respectively. Cognitive performance was assessed with a focus on executive function and verbal memory, areas that have been demonstrated to be relevant in BD. ${ }^{18}$ Executive function was evaluated using the Stroop Color and Word Test ${ }^{19}$ and the standard score of the Wisconsin Card Sorting Test (WCST). ${ }^{20}$ Verbal memory was measured using the California Verbal Learning Test (CVLT). ${ }^{21}$

Psychosocial functioning was assessed using the Functioning Assessment Short Test (FAST), a four-point Likerttype scale containing 24 items whose scores may range from 0 to 72; higher scores indicate poorer functional status. ${ }^{22}$ The scale covers six specific areas of psychosocial functioning: autonomy, occupational functioning, cognitive functioning, financial issues, interpersonal relationships, and leisure time. The FAST has been validated for use both in the Brazilian population ${ }^{23}$ and in patients with $\mathrm{BD},{ }^{22}$ and has been reported to be sensitive to change. ${ }^{24,25}$

\section{Statistics}

Statistical analyses were performed using the SPSS version 19.0. The three groups (patients, siblings, and healthy controls) were compared with regard to clinical and sociodemographic characteristics using either analysis of variance or the chi-square test, as appropriate. Multivariate analysis of covariance (MANCOVA) was performed to examine differences in neuropsychological variables between groups. Cognitive variables were used as dependent variables, mood symptoms (HDRS and YMRS results) as covariates, and the group was the main factor. Comparisons showing significant effects were further analyzed using MANCOVA followed by Tukey's honestly significant difference post-hoc tests. 
Pearson's correlation was used to evaluate the relationship between functioning, cognitive variables, and clinical variables in the group of patients with BD. A linear regression model was used to identify the best predictors of psychosocial functioning; all clinical variables showing correlation with FAST scores were tested. All analyses were two-tailed. Significance was set at $p<0.050$.

\section{Results}

Thirty-six patients with BD, 39 unaffected siblings, and 44 healthy subjects were included in the study. Sociodemographic variables and mood rating scale scores are shown in Table 1. Variables, including gender, age, and years of education were not significantly different between patients, siblings, and control groups.

Patients, siblings, and healthy controls all showed very low sub-threshold symptoms, as indicated by mood rating scale scores. However, there were statistical differences in depressive and manic symptom scores between patients and healthy controls $(p<0.010)$ and between siblings and healthy controls $(p<0.010)$. As expected, patients with BD showed higher FAST total scores $(23.91 \pm 11.35)$ when compared to both healthy controls

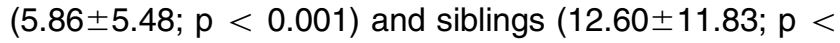
$0.001)$. Siblings and healthy controls also showed statistical differences in FAST total scores $(p=0.008)$.

Patients also showed higher functional impairment than healthy controls when FAST domains were analyzed separately (all $p<0.004$ ). When compared with siblings, patients showed higher functional impairment in the autonomy ( $p \leqslant 0.001)$, occupational $(p \leqslant 0.001)$, cognition ( $p=0.004)$, financial $(p=0.030)$, and interpersonal relationships $(p=0.010)$ domains. There were also statistical differences between siblings and healthy controls, with siblings showing higher impairment in cognition ( $p=0.010)$, interpersonal relationships $(p=0.003)$, and leisure time $(p=0.040)$. FAST scores obtained for all three groups are shown in Table 2.

Because the groups differed in terms of HDRS scores, MANCOVA was used to control for potential confounders, using the group as the main factor. The analysis revealed significant differences in functioning between the groups, even when HDRS was used as a covariate (Pillai's $F=$ 3.620 , degrees of freedom [df] $=2, p \leqslant 0.001$ ).

Neuropsychological results are shown in Table 3. MANCOVA (Pillai's $F=1.481$, df $=2, p<0.001$ ) was performed for the main effect and revealed significant differences in cognitive performance between the groups. Patients performed worse than both healthy controls and siblings on all CVLT sub-tests (all $p<0.010$ ). Executive function showed significant differences between patients and healthy controls in the number of correctly completed categories on WCST $(p=0.030)$. The same pattern was found when HDRS and YMRS scores were used as covariates in MANCOVA. Medications currently in use by the patients who performed neuropsychological tests are shown in Table 1.

Correlations were found between CVLT long delayed free recall and HDRS ( $r=-0.44, p=0.020)$, and between CVLT total recall and HDRS $(r=-0.51, p \leqslant 0.010)$. There were also significant positive correlations between FAST total scores and HDRS $(r=0.65, p<0.001)$, number of previous depressive episodes $(r=0.55, p=0.002)$, number of previous manic episodes $(r=0.63, p<0.001)$, and number of previous hospitalizations $(r=0.38, p=0.03)$.

Finally, in the linear regression model, HDRS scores, number of depressive episodes, number of psychiatric hospitalizations, and number of manic episodes were entered simultaneously as predictors into a regression equation using FAST total scores as the dependent measure. Only HDRS scores remained a significant predictor of FAST total scores $(B=1.81$, standard error $=0.57, p=0.004)$.

Table 1 Demographic and clinical characteristics of patients with BD, unaffected siblings, and healthy controls

\begin{tabular}{|c|c|c|c|c|}
\hline Characteristics & Patients $(n=36)$ & Siblings $(n=39)$ & Controls $(n=44)$ & $p$-value \\
\hline $\begin{array}{l}\text { Age (years)* } \\
\text { HDRS score* } \\
\text { YMRS score* }\end{array}$ & $\begin{array}{c}47.25(10.16) \\
4.11(2.94) \\
0.94(1.53)\end{array}$ & $\begin{array}{c}49.76(15.02) \\
3.59(3.50) \\
0.10(1.53)\end{array}$ & $\begin{array}{c}45.84(12.15) \\
0.70(1.15) \\
0.09(0.36)\end{array}$ & $\begin{array}{c}0.359 \\
>0.001 \\
0.001\end{array}$ \\
\hline $\begin{array}{l}\text { Sex }^{\dagger} \\
\quad \text { Male } \\
\text { Female }\end{array}$ & $\begin{array}{c}9(25.00) \\
27(75.00)\end{array}$ & $\begin{array}{l}12(30.76) \\
27(69.23)\end{array}$ & $\begin{array}{l}20(45.45) \\
24(54.54)\end{array}$ & 0.134 \\
\hline Illness duration & $19.78(11.54)$ & & & \\
\hline $\begin{array}{l}\text { Current medications }{ }^{\S} \\
\text { Lithium } \\
\text { Valproic acid } \\
\text { Typical antipsychotics } \\
\text { Atypical antipsychotics } \\
\text { Antidepressants } \\
\text { Benzodiazepines }\end{array}$ & $\begin{aligned} 14 & (58.33) \\
12 & (50.00) \\
2 & (8.34) \\
12 & (50.01) \\
2 & (8.34) \\
3 & (12.51)\end{aligned}$ & & & \\
\hline
\end{tabular}

\footnotetext{
Data presented as mean (standard deviation) or $\mathrm{n}(\%)$.

$\mathrm{BD}=$ bipolar disorder; HDRS = Hamilton Depression Rating Scale; YMRS = Young Mania Rating Scale.

* p-value by ANOVA.

p-value by chi-square test.

$\$$ Illness duration calculated considering age at diagnosis of BD.

${ }^{\S}$ Medications currently in use by patients who completed the neuropsychological battery $(n=21)$.
} 
Table 2 Psychosocial functioning as assessed by specific FAST domains in patients with BP, unaffected siblings, and healthy controls

\begin{tabular}{|c|c|c|c|c|}
\hline FAST & Patients* $(n=33)$ & Siblings $^{\dagger}(n=35)$ & Controls $(n=43)$ & F/p-value \\
\hline Total score & $23.91(11.35)$ & $12.60(11.83)$ & $5.86(5.48)$ & $32.01 /<0.001$ \\
\hline Autonomy & $3.41(2.85)$ & $1.26(2.47)$ & $0.63(1.04)$ & $15.80 /<0.001$ \\
\hline Occupational & $5.38(4.79)$ & $1.69(2.55)$ & $0.86(1.39)$ & $21.20 /<0.001$ \\
\hline Cognitive & 6.47 (3.49) & $4.11(3.11)$ & $2.14(2.29)$ & $19.83 /<0.001$ \\
\hline Financial & $1.94(2.09)$ & $0.97(1.58)$ & $0.53(0.85)$ & $7.81 / 0.001$ \\
\hline Interpersonal relationships & $4.69(3.05)$ & $2.86(3.37)$ & $0.79(1.47)$ & $19.72 /<0.001$ \\
\hline Leisure time & $2.03(1.59)$ & $1.71(1.84)$ & $0.91(0.97)$ & $5.89 / 0.004$ \\
\hline
\end{tabular}

Data presented as mean (standard deviation).

$\mathrm{BD}=$ bipolar disorder; FAST $=$ Functioning Assessment Short Test.

* Patients showed higher functional impairment than healthy controls (all $p<0.004$ ), higher impairment than unaffected siblings in autonomy

$(p=0.001)$, occupational functioning $(p<0.001)$, cognition $(p=0.004)$, financial $(p=0.03)$, and interpersonal relationships $(p=0.01)$.

Unaffected siblings showed higher impairment than healthy controls in cognition $(p=0.01)$, interpersonal relationships $(p=0.003)$, and

leisure time $(p=0.04)$.

${ }^{\ddagger} \mathrm{p}$-value by ANOVA.

\section{Discussion}

To the authors' knowledge, this is the first study to compare cognitive performance and psychosocial functioning between patients with BD, unaffected siblings, and healthy controls. The findings of this research can be summarized as follows: 1) there were significant differences in memory tasks and executive function between patients and healthy controls, but not between siblings and controls; 2) patients showed higher functional impairment according to FAST scores compared to both healthy controls (all domains) and unaffected siblings (some domains); 3) there were statistical differences between siblings and healthy controls in FAST total scores and in specific domains of the scale, namely, cognition, interpersonal relationships, and leisure time.

With regard to patients and healthy controls, our findings are consistent with previous studies that have shown higher cognitive impairment, in particular in executive function and verbal memory, in remitted patients with BD compared to controls, ${ }^{7,10,11}$ as well as greater functioning impairment in patients vs. healthy controls. ${ }^{3}$

However, our study also showed poorer psychosocial functioning in unaffected siblings of patients with BD compared to healthy controls, as measured by the FAST. To our knowledge, this is the first study to evaluate psychosocial functioning in siblings using this instrument. Linnen et al., ${ }^{26}$ Ellenbogen et al., ${ }^{27}$ and Ostiguy et al. ${ }^{28}$ investigated psychosocial functioning in the offspring of patients with $\mathrm{BD}$, but those authors evaluated specifically interpersonal relationships; all three studies found some degree of impairment in the domain assessed. Bella et al. $^{29}$ evaluated other areas in addition to interpersonal relationships in children of patients with $\mathrm{BD}$, and also found impairment. However, we could not find other studies assessing psychosocial functioning in unaffected siblings of patients with BD.

The FAST scores obtained in the present study placed siblings in an intermediate position between patients with $\mathrm{BD}$ and healthy controls, suggesting that psychosocial functioning could be either an endophenotypic trait or a sign of vulnerability and/or resilience in this population. Rosa et al. $^{3}$ found higher FAST scores in late-stage BD patients than in early-stage patients, and suggested that FAST scores could have the potential to distinguish between patients in early vs. late stages of BD. We could speculate that the results obtained with siblings in the present study are consistent with the staging model of $B D$ - siblings might have a so-called latent-stage $B D,{ }^{30}$ as a

Table 3 Cognitive performance of patients with BD, unaffected siblings, and healthy controls

\begin{tabular}{|c|c|c|c|c|c|}
\hline & Patients $(n=21)$ & Siblings $(n=14)$ & Controls $(n=22)$ & ANOVA F/p-value & Post-hoc \\
\hline CVLT total recall & $42.61(10.57)$ & $50.56(8.07)$ & $51.65(10.75)$ & $5.45 / 0.007$ & $\mathrm{P}<\mathrm{HC}, \mathrm{S}^{*}$ \\
\hline ayed free recall 1 & $8.22(2.96)$ & 9 (2.11) & (2.65) & $9.40 /<0.001$ & $\mathrm{P}<\mathrm{HC}, \mathrm{S}^{\dagger}$ \\
\hline CVLT long delayed free recall 2 & $8.78(2.84)$ & $11.11(2.65)$ & $10.11(2.97)$ & $5.63 / 0.006$ & $\mathrm{P}<\mathrm{HC}, \mathrm{S}^{\ddagger}$ \\
\hline CVLT short delayed cued recall 1 & $9.43(2.46)$ & $11.50(1.65)$ & $11.65(2.55)$ & $6.45 / 0.003$ & $\mathrm{P}<\mathrm{HC}, \mathrm{S}^{\S}$ \\
\hline CVLT long delayed cued recall 2 & $9.22(2.71)$ & $11.78(2.13)$ & $11.70(2.40)$ & $7.77 / 0.001$ & $\mathrm{P}<\mathrm{HC}, \mathrm{S}^{\|}$ \\
\hline Stroop Color and Word Test & $107.93(69.13)$ & $82.77(19.05)$ & $79.60(56.81)$ & $1.80 / 0.173$ & $\mathrm{P}=\mathrm{HC}=\mathrm{S}$ \\
\hline WCST perseverative errors & $83.61(13.93)$ & $80.06(15.14)$ & $88.13(16.30)$ & $1.46 / 0.240$ & $\mathrm{P}=\mathrm{S}=\mathrm{HC}$ \\
\hline WCST number of correctly completed categories & $2.70(1.29)$ & $2.89(1.53)$ & $3.74(1.35)$ & $3.62 / 0.033$ & $\mathrm{P}<\mathrm{HC}^{\bullet}$ \\
\hline
\end{tabular}

Data presented as mean (standard deviation).

$\mathrm{BD}=$ bipolar disorder; CVLT = California Verbal Learning Test; $\mathrm{HC}=$ healthy controls; $\mathrm{P}=$ patients; $\mathrm{S}=$ siblings; WCST = Wisconsin Card Sorting Test.

$* p \leqslant 0.01$ between patients and healthy controls, $p=0.01$ between patients and unaffected siblings.

$p \leqslant 0.01$ between patients and unaffected siblings, $p<0.01$ between patients and healthy controls.

$\ddagger p \leqslant 0.01$ between patients and healthy controls, $p \leqslant 0.01$ between patients and unaffected siblings.

${ }_{1} p \leqslant 0.01$ between patients and unaffected siblings, $p \leqslant 0.01$ between patients and healthy controls.

$\| p \leqslant 0.01$ between patients and unaffected siblings, $p<0.01$ between patients and healthy controls.

$p=0.03$ between patients and healthy controls. 
result of the biological underpinnings of $\mathrm{BD}$ that they share. Conversely, it is also possible that the siblings selected for the present study had a high level of resilience, as they remained without psychiatric diagnosis despite the genetic and environmental factors shared with the patients. Of note, the mean age of siblings in our sample was 45.6 years, which is beyond the first peak age of $\mathrm{BD}$ onset (BD type I has two peaks of onset age, the first at 15-24 years and the second at 45-54 years). ${ }^{31}$

Neurocognitive deficits in patients with BD, an established part of the disorder, have been strongly correlated with poor functioning ${ }^{6-8}$ and with other clinical factors, such as disease severity, chronicity, and residual symptoms. ${ }^{9,32}$ Another study has shown that patients with multiple-episode BD have poorer functioning, especially in the FAST autonomy, occupational, interpersonal relationships, and leisure time domains, compared to first-episode patients. ${ }^{33}$ Finally, Forcada et al. ${ }^{8}$ showed that IQ, total white matter volume, and a predominantly depressive illness course were independently associated with functional outcomes in patients with $\mathrm{BD}$, but not in their relatives.

In line with the findings described above, the present study found poor cognition and functioning in patients with BD. CVLT parameters in patients were different from those obtained for controls and also for siblings, reinforcing the effect of the disease on cognitive outcomes. This finding highlights the damage caused by number of episodes and number of hospitalizations, as suggested by Strakowski et al., ${ }^{34}$ who have shown abnormalities in neural circuits involved in mood regulation and cognition in multiple-episode patients. In the present study, CVLT, Stroop Color and Word Test, and WCST results were similar in siblings and controls.

There are studies reporting significant impairment of verbal working memory in siblings of patients with $B D$ compared to healthy controls. ${ }^{35,36}$ Similar results have been found in comparisons between first-degree relatives (siblings, parents) and healthy controls. ${ }^{37,38}$ Conversely, other authors have reported negative results for verbal learning, with no differences between first-degree relatives of patients with BD and healthy control groups. ${ }^{39,40}$ However, comparison across those studies is limited, as samples are not homogenous with regard to the type and age of first-degree relatives included (siblings, offspring, and parents).

Kulkarni et al. ${ }^{35}$ assessed sample similar to ours, i.e., middle-aged siblings and controls. In that study, unaffected siblings performed poorly on the Tower of London Test (a planning test) and on Rey's Auditory Verbal Learning Test, but there were no significant differences between siblings and healthy controls on the Stroop Color and Word Test, the WCST, and the lowa Gambling Task (IGT). Nehra et al. $^{36}$ also reported poor performance of middle-aged unaffected siblings of patients with $\mathrm{BD}$ in verbal learning tests, assessed using the Hopkins Verbal Learning Test-Revised. Again, these controversial findings reported for unaffected relatives may be explained by heterogeneous samples (type and age of relative assessed) and by methodological differences, e.g., in the cognitive tests employed. Moreover, in our study, only a sub-group of participants (smaller sample size) underwent the neurocognitive test battery, reducing the power to detect subtler impairments - which we would expect to be the case with siblings, considering their intermediate position between patients and controls in terms of functional scores. Further studies are warranted to assess cognitive reserve in siblings of patients with $\mathrm{BD}$, given the apparently relevant role played by this variable in cognitive impairment in BD. ${ }^{32}$

Our findings need to be considered in light of two major limitations: first, our small sample size; second, the crosssectional design of our study. It would be interesting to see the effects of a larger sample and of a longitudinal design on the results here reported.

In conclusion, the present study suggests that unaffected siblings of patients with BD may have poorer functional performance when compared to healthy controls, probably as a result of the genetic background that they share with the patients. The use of FAST scores may contribute to the development of markers in at-risk populations, and may be considered as an endophenotypic trait. It remains to be known whether the impairment of psychosocial functioning found in unaffected siblings has any effect on their quality of life. Further prospective studies are needed to improve our knowledge in this area and help identify endophenotypes.

\section{Acknowledgements}

This work was supported by grants from the Instituto Nacional de Ciência e Tecnologia Translacional em Medicina (INCT-TM), Conselho Nacional de Desenvolvimento Científico e Tecnológico (CNPq), and Fundo de Incentivo è Pesquisa-Hospital de Clínicas de Porto Alegre (FIPE-HCPA). MK has received research grants from CNPq-INCT-TM and CNPq-Universal. EV has received research grants from the Spanish Ministry of Science and Innovation, the Stanley Medical Research Institute (SMRI), and The 7th Framework Program of the European Union. FK has received grants/research support from CNPq, Coordenação de Aperfeiçoamento de Pessoal de Nível Superior (CAPES), the National Alliance for Research in Schizophrenia and Affective Disorders (NARSAD), and SMRI. ARR has received support from L'Oréal Brasil, Academia Brasileira de Ciências, and the UNESCO National Commission For Women in Science. MKS has received research grants and/or speaking fees from CNPq/INCT-TM, CNPq/Universal, CAPES, SMRI, NARSAD, and Fundo de Incentivo è Pesquisa - Hospital de Clínicas de Porto Alegre (FIPE-HCPA).

\section{Disclosure}

MKS has received speaking fees Eli Lilly. FK has received grants/research support from AstraZeneca, Eli Lilly, Janssen-Cilag, and Servier; has been a member of the speakers' board for AstraZeneca, Eli Lilly, Janssen, and Servier; and has served as a consultant for Servier. EV has served as consultant, advisor, and/or speaker for Almirall, AstraZeneca, Bristol-Myers Squibb, Eli Lilly, Forest Research, Geodon Richter, GlaxoSmithKline, 
Janssen-Cilag, Jazz, Lundbeck, Merck, Novartis, Organon, Otsuka, Pfizer, Sanofi-Aventis, Servier, Solvay, Schering-Plough, Takeda, United Biosource, and Wyeth. The other authors report no conflicts of interest.

\section{References}

1 Antypa N, Serretti A. Family history of a mood disorder indicates a more severe bipolar disorder. J Affect Disord. 2014;156:178-86.

2 Kessler RC, McGonagle KA, Zhao S, Nelson CB, Hughes M, Eshleman S, et al. Lifetime and 12-month prevalence of DSM-III-R psychiatric disorders in the United States. Results from the National Comorbidity Survey. Arch Gen Psychiatry. 1994;51:8-19.

3 Rosa AR, Magalhães PV, Czepeilewski L, Sulzbach MV, Goi PD, Vieta E, et al. Clinical staging in bipolar disorder: focus on cognition and functioning. J Clin Psychiatry. 2014;75:e450-6.

4 Gonzalez-Pinto AM, Dardennes R, de Zélicourt M, López P, Oliveros RG, Vieta $E$, et al. In-patient care costs of patients with bipolar I disorder: a comparison between two European centers. J Affect Disord. 2010;121:152-5.

5 Tohen M, Hennen J, Zarate CM Jr, Baldessarini RJ, Strakowski SM, Stoll AL, et al. Two-year syndromal and functional recovery in 219 cases of first-episode major affective disorder with psychotic features. Am J Psychiatry. 2000;157:220-8.

6 Bonnín CM, Martinez-Aran A, Torrent C, Pacchiarotti I, Rosa AR, Franco $\mathrm{C}$, et al. Clinical and neurocognitive predictors of functional outcome in bipolar euthymic patients: a long-term, follow-up study $J$ Affect Disord. 2010;121:156-60.

7 Martinez-Aran A, Vieta E, Torrent C, Sanchez-Moreno J, Goikolea $\mathrm{JM}$, Salamero M, et al. Functional outcome in bipolar disorder: the role of clinical and cognitive factors. Bipolar Disord. 2007:9:103-13.

8 Forcada I, Papachristou E, Mur M, Christodoulou T, Jogia J, Reichenberg $A$, et al. The impact of general intellectual ability and white matter volume on the functional outcome of patients with Bipolar Disorder and their relatives. J Affect Disord. 2011;130:413-20.

9 Mur M, Portella MJ, Martinez-Aran A, Pifarre J, Vieta E. Influence of clinical and neuropsychological variables on the psychosocial and occupational outcome of remitted bipolar patients. Psychopathology. 2009;42:148-56.

10 Torres IJ, Boudreau VG, Yatham LN. Neuropsychological functioning in euthymic bipolar disorder: a meta-analysis. Acta Psychiatr Scand Suppl. 2007;434:17-26.

11 Mackala SA, Torres IJ, Kozicky J, Michalak EE, Yatham LN. Cognitive performance and quality of life early in the course of bipolar disorder. J Affect Disord. 2014;168:119-24.

12 Hill SK, Reilly JL, Harris MS, Rosen C, Marvin RW, Deleon O, et al. A comparison of neuropsychological dysfunction in first-episode psychosis patients with unipolar depression, bipolar disorder, and schizophrenia. Schizophr Res. 2009:113:167-75.

13 Torres IJ, DeFreitas VG, DeFreitas CM, Kauer-Sant'Anna M, Bond DJ, Honer WG, et al. Neurocognitive functioning in patients with bipolar I disorder recently recovered from a first manic episode. J Clin Psychiatry. 2010;71:1234-42.

14 Arts B, Jabben N, Krabbendam L, Van Os J. Meta-analyses of cognitive functioning in euthymic bipolar patients and their first-degree relatives. Psychol Med. 2008;38:771-85.

15 Balanzá-Martínez V, Rubio C, Selva-Vera G, Martinez-Aran A, Sánchez-Moreno J, Salazar-Fraile J, et al. Neurocognitive endophenotypes (endophenocognitypes) from studies of relatives of bipolar disorder subjects: a systematic review. Neurosci Biobehav Rev. 2008;32:1426-38.

16 Bora E, Yucel M, Pantelis C. Cognitive endophenotypes of bipolar disorder: A meta-analysis of neuropsychological deficits in euthymic patients and their first-degree relatives. J Affect Disord. 2009;113:1-20.

17 Fusar-Poli $\mathrm{P}$, Howes $\mathrm{O}$, Bechdolf A, Borgwardt S. Mapping vulnerability to bipolar disorder: a systematic review and meta-analysis of neuroimaging studies. J Psychiatry Neurosci. 2012;37:170-84.

18 Yatham LN, Torres IJ, Malhi GS, Frangou S, Glahn DC, Bearden CE, et al. The International Society for Bipolar Disorders-Battery for Assessment of Neurocognition (ISBD-BANC). Bipolar Disord. 2010;12:351-63.

19 Golden CJ. Stroop Color and Word Test. Chicago: Stoelting; 1978.
20 Heaton RK, Chelune GJ, Talley JL, Kay GG, Curtiss G. Wisconsin card sort test manual: revised and expanded. Odessa: Psychological Assessment Resources; 1993.

21 Delis DC, Kramer JH, Kaplan E, Ober BA. California verbal learning test manual. 2nd ed.San Antonio: Psychological Corporation; 2000.

22 Rosa AR, Sanchez-Moreno J, Martínez-Aran A, Salamero M, Torrent $C$, Reinares $M$, et al. Validity and reliability of the Functioning Assessment Short Test (FAST) in bipolar disorder. Clin Pract Epidemiol Ment Health. 2007;3:5-5.

23 Cacilhas AA, Magalhães PV, Ceresér KM, Walz JC, Weyne F, Rosa AR, et al. Validity of a short disability test (FAST) in Brazilian outpatients with bipolar disorder. Value Health. 2009;12:624-7.

24 Rosa AR, Reinares M, Amann B, Popovic D, Franco C, Comes M, et al. Six-month functional outcome of a bipolar disorder cohort in the context of a specialized-care program. Bipolar Disord. 2011;13:679-86.

25 Torrent C, Bonnin Cdel M, Martínez-Arán A, Valle J, Amann BL, González-Pinto A, et al. Efficacy of functional remediation in bipolar disorder: a multicenter randomized controlled study. Am J Psychiatry. 2013;170:852-9.

26 Linnen AM, aan het Rot M, Ellenbogen MA, Young SN. Interpersonal functioning in adolescent offspring of parents with bipolar disorder. $\mathrm{J}$ Affect Disord. 2009;114:122-30.

27 Ellenbogen MA, Linnen AM, Santo JB, aan het Rot M, Hodgins S, Young SN. Salivary cortisol and interpersonal functioning: an eventcontingent recording study in the offspring of parents with bipolar disorder. Psychoneuroendocrinology. 2013;38:997-1006.

28 Ostiguy CS, Ellenbogen MA, Hodgins S. Personality of parents with bipolar disorder and interpersonal functioning among their offspring: a prospective 10-year study. Dev Psychopathol. 2012;24:573-87.

29 Bella T, Goldstein T, Axelson D, Obreja M, Monk K, Hickey MB, et al. Psychosocial functioning in offspring of parents with bipolar disorder. J Affect Disord. 2011;133:204-11.

30 Kapczinski F, Dias VV, Kauer-Sant'Anna M, Frey BN, Grassi-Oliveira $\mathrm{R}$, Colom $\mathrm{F}$, et al. Clinical implications of a staging model for bipolar disorders. Expert Rev Neurother. 2009:9:957-66.

31 Kroon JS, Wohlfarth TD, Dieleman J, Sutterland AL, Storosum JG, Denys $\mathrm{D}$, et al. Incidence rates and risk factors of bipolar disorder in the general population: a population-based cohort study. Bipolar Disord. 2013;15:306-13.

32 Forcada I, Mur M, Mora E, Vieta E, Bartrés-Faz D, Portella MJ. The influence of cognitive reserve on psychosocial and neuropsychological functioning in bipolar disorder. Eur Neuropsychopharmacol. 2015;25:214-22.

33 Rosa AR, González-Ortega I, González-Pinto A, Echeburúa E, Comes M, Martínez-Àran A, et al. One-year psychosocial functioning in patients in the early vs. late stage of bipolar disorder. Acta Psychiatr Scand. 2012;125:335-41.

34 Strakowski SM, Delbello MP, Zimmerman ME, Getz GE, Mills NP, Ret J, et al. Ventricular and periventricular structural volumes in first versus multiple-episode bipolar disorder. Am J Psychiatry. 2002;159:1841-7.

35 Kulkarni S, Jain S, Janardhan Reddy YC, Kumar KJ, Kandavel T. Impairment of verbal learning and memory and executive function in unaffected siblings of probands with bipolar disorder. Bipolar Disord. 2010;12:647-56.

36 Nehra R, Grover S, Sharma S, Sharma A, Sarkar S. Neuro-cognitive functioning in unaffected siblings of patients with bipolar disorder: comparison with bipolar patients and healthy controls. Indian J Psychiatry. 2014;56:283-8.

37 Bora E, Vahip S, Akdeniz F, ilerisoy H, Aldemir E, Alkan M. Executive and verbal working memory dysfunction in first-degree relatives of patients with bipolar disorder. Psychiatry Res. 2008;161:318-24.

38 Glahn DC, Almasy L, Barguil M, Hare E, Peralta JM, Kent Jr JW, et al. Neurocognitive endophenotypes for bipolar disorder identified in multiplex multigenerational families. Arch Gen Psychiatry. 2010;67:168-77.

39 Civil Arslan F, Tiryaki A, Ozkorumak E. A comparison of euthymic bipolar patients with unaffected first-degree relatives and healthy controls in terms of neuropsychological functions. Int J Psychiatry Clin Pract. 2014;18:208-14.

40 Clark L, Sarna A, Goodwin GM. Impairment of executive function but not memory in first-degree relatives of patients with bipolar I disorder and in euthymic patients with unipolar depression. Am J Psychiatry. 2005;162:1980-2. 